\title{
On a Curvature Condition that Implies a Cohomology Injectivity Theorem of Kollár-Skoda Type
}

By

\author{
Takeo OHSAWA*
}

\begin{abstract}
The curvature condition for the singular Hermitian metric in a generalized $L^{2}$ extension theorem on complex manifolds implies also a cohomology injectivity theorem in certain circumstances. This shows that the curvature condition is still available for the extension theorem in more general situations than before.
\end{abstract}

\section{$\S 1 . \quad$ Introduction}

Let $M$ be a complex manifold, let $S \subset M$ be a complex analytic subset, and let $E \rightarrow M$ be a holomorphic vector bundle. An extension problem in complex analysis asks for the conditions on the triple $(M, S, E)$ under which the restriction map from the set of holomorphic sections of $E$ over $M$ to that over $S$ is surjective. It is known from a celebrated work of $\mathrm{H}$. Cartan [C] that this extension problem is solvable for any pair if $M$ is a Stein manifold. More refined extendability criterion with respect to the sections with growth conditions has been studied from various viewpoints. As for the $L^{2}$ spaces of holomorphic sections, results have been obtained in [O-T], [M], [D-2]. (See also $[\mathrm{O}-2,4,5,6]$.$) These are derived by exploiting a "twisted" variant of Nakano's$ identity and described, under natural assumptions on $M$ for smooth $S$, in terms of the curvature property of $(M, S, E)$ (cf. [O-5, Theorem 4]).

Communicated by K. Saito. Received September 9, 2003. Revised December 10, 2003, February 6, 2004.

2000 Mathematics Subject Classification(s): Primary 32W05, Secondary 32T05.

*Graduate School of Mathematics, Nagoya University, Chikusaku Furocho 464-8602

Nagoya Japan.

e-mail: ohsawa@math.nagoya-u.ac.jp

(C) 2005 Research Institute for Mathematical Sciences, Kyoto University. All rights reserved. 
On the other hand, if the singular locus of $S$ is nonempty, it may occur that some bounded holomorphic functions are not $L^{2}$ extendable with respect to a finite measure (cf. [D-M]). Even the presence of an isolated singularity in $S$ changes the situation in an essential way (cf. $\S 4$ ).

Nevertheless, in some extension problem arising from the classification theory of algebraic varieties, the curvature condition which arose in the $L^{2}$ extension theorem is still available to give a sufficient condition for the extendability of certain cohomology classes from singular subvarieties. This can be observed, for instance, from the works of Tankeev [Tn], Kollár [K], Enoki [E] and Takegoshi $[\mathrm{Tg}]$.

The purpose of the present article is to pursue this point by establishing the following.

Theorem 1.1. Let $M$ be a weakly 1-complete Kähler manifold, let $(E, h)$ be a Hermitian holomorphic vector bundle over $M$, and let $(L, b)$ be a Hermitian holomorphic line bundle over $M$. Suppose that the curvature forms $\Theta_{h}, \Theta_{b}$ of $h, b$ satisfy $\Theta_{h} \geq 0$ and $\Theta_{h}-\varepsilon \operatorname{Id}_{E} \otimes \Theta_{b} \geq 0$ for some $\varepsilon>0$, both in the sense of Nakano. Then, for any nonzero holomorphic section s of $L$, the kernel of the multiplication homomorphism $s: H^{q}\left(M, K_{M} \otimes E\right) \rightarrow H^{q}\left(M, K_{M} \otimes E \otimes L\right)$ is contained in the closure of zero for any $q$. Here $K_{M}$ denotes the canonical line bundle of $M$.

Since the analytic cohomology groups of holomorphically convex manifolds are known to be Hausdorff in virtue of Grauert's direct image theorem and Remmert's reduction theorem, the following is an immediate consequence of Theorem 1.1.

Corollary 1.1. Under the situation of Theorem 1, suppose moreover that $M$ is holomorphically convex. Then the restriction maps

$$
H^{q}\left(M, K_{M} \otimes E \otimes L\right) \rightarrow H^{q}\left(s^{-1}(0), K_{M} \otimes E \otimes L\right)
$$

are surjective for any $q$. Here $s^{-1}(0)$ is equipped with the structure of complex analytic space with structure sheaf $\mathcal{O}_{M} / s \mathcal{O}_{M}\left(L^{-1}\right)$.

As for Theorem 1.1, a general result of this type was first established by Kollár $[\mathrm{K}]$ for the nonsingular projective varieties.

It was proved in $[\mathrm{Tg}]$ that Theorem 1.1 is true if $E=E_{0} \otimes F^{k}(k \geq 1)$ and $L=F^{j}(j \geq 1)$ for some Nakano semipositive vector bundle $E_{0}$ and a semipositive line bundle F. Thus Theorem 1.1 strengthens Takegoshi's result by weakening the curvature assumptions. 
Since the corollary is an extension theorem from divisors, it is natural to expect its generalization to higher codimensional cases. Indeed, by a similar argument we are able to prove the following Skoda type injectivity theorem (cf. $[\mathrm{Sk}]$ ).

Theorem 1.2. Let $M$ be a weakly 1-complete Kähler manifold, let $(E, h)$ be a Hermitian holomorphic vector bundle over $M$, and let $\mathcal{I}$ be a coherent ideal sheaf of $\mathcal{O}_{M}$. Suppose that there exists a $C^{2}$ function $\sigma: M \rightarrow[0, \infty)$ with $\sigma^{-1}(0)=\operatorname{supp} \mathcal{O}_{M} / \mathcal{I}$ such that

$$
\Theta_{h}+(1+t) \operatorname{Id}_{E} \otimes \partial \bar{\partial} \log \sigma \geq 0
$$

holds on $M \backslash \sigma^{-1}(0)$ for $0 \leq t<\varepsilon$ for some $\varepsilon>0$ and that

$$
\begin{gathered}
\mathcal{I}_{x}=\left\{f_{x} \mid f\right. \text { is holomorphic on some coordinate neighbourhood } \\
\left.U \ni x \text { and satisfies that } \int_{U}|f|^{2} / \sigma<\infty\right\} .
\end{gathered}
$$

Then the kernel of the homomorphism

$$
H^{q}\left(M, K_{M} \otimes E \otimes \mathcal{I}\right) \rightarrow H^{q}\left(M, K_{M} \otimes E\right)
$$

is contained in the closure of zero for any $q \geq 0$. Here we identify $E$ with $\mathcal{O}_{M}(E)$ for simplicity.

Restricting ourselves to holomorphically convex manifolds we obtain

Theorem 1.3. If $M$ is a holomorphically convex Kähler manifold and $E$ is a holomorphic line bundle over $M$ equipped with a singular Hermitian metric $h=h_{0} e^{-\psi}$, with $h_{0} \in C^{\infty}$ and $\psi \in L_{\text {loc }}^{1}$, such that $\Theta_{h} \geq 0$ and $\Theta_{h}-$ $\varepsilon \operatorname{Id}_{E} \otimes \partial \bar{\partial} \psi \geq 0$ for some $\varepsilon>0$, in the sense of current, then the restriction map

$$
H^{q}\left(M, K_{M} \otimes E\right) \rightarrow H^{q}\left(M, K_{M} \otimes E \otimes\left(\mathcal{O}_{M} / I(h)\right)\right)
$$

is surjective for any $q$, where $I(h)$ denotes the multiplier ideal sheaf of $h$.

The reader will be reffered to [D-2, Remark 3.2 and Theorem 4.1, Step 4.6] for the supplementary argument to take care of the possible irregularity of $\psi$. We note that Theorem 1.3 is a natural generalization of Nadel's vanishing theorem [Nd], also in Skoda's spirit. In $\S 4$ we shall give its application to the analytic Zariski decomposition. It will be shown that, if the canonical bundle of $M$ admits analytic Zariski decomposition $b e^{-\psi}$ with certain curvature property 
(see $\S 4$ ), then the (ideal) support of the multiplier ideal sheaf of $b e^{-\psi}$ does not contain any isolated point.

The author would like to thank the referee for valuable criticism.

\section{§2. Preliminaries}

Let $(M, g)$ be a connected complete Kähler manifold of dimension $n$, let $C_{0}^{p, q}(M)$ be the space of $C^{\infty}(p, q)$-forms on $M$ with compact support, and let $\bar{\partial}$ be the complex exterior differentiation of type $(0,1)$ acting on the space of $(p, q)$-currents. Let $(E, h)$ be a Hermitian holomorphic vector bundle over $M$. By $C_{0}^{p, q}(M, E)\left(\operatorname{resp} . K^{p, q}(M, E)\right)$ we denote the space of $E$-valued $C^{\infty}(p, q)$ forms with compact support (resp. that of $E$-valued $(p, q)$-currents). Metrics are supposed to be $C^{\infty}$, or differentiable to any necessary order.

For $u, v \in C_{0}^{p, q}(M, E)$, let $(u, v)\left(=(u, v)_{g, h}\right)$ be the inner product of $u$ and $v$ defined by the integral

$$
(u, v)=\int_{M}\langle u, v\rangle d V
$$

where $\langle u, v\rangle$ denotes the pointwise inner product of $u$ and $v$ with respect to $g$ and $h$, and $d V$ the volume form with respect to $g$. Then we define the $L^{2}$ norm $\|u\|$ of $u$ by $\|u\|^{2}\left(=\|u\|_{g, h}^{2}\right)=(u, u)$. Let $\omega\left(=\omega_{g}\right)$ be the fundamental form of $g$. By $e(\omega)$ we denote the exterior multiplication by $\omega$. The adjoint of $e(\omega)$ with respect to $\langle$,$\rangle will be denoted by \Lambda$. Let $*$ be the star operator with respect to $g$. Then, identifying the fiber metric $h$ naturally with a section of $\operatorname{Hom}\left(E, \bar{E}^{*}\right), \bar{E}^{*}$ being the dual of the complex conjugate $\bar{E}$ of $E$, and letting $h$ operate on $C_{0}^{p, q}(M, E)$ coefficientwise, we have

$$
(u, v)=\int_{M} h(u) \wedge \bar{*} v
$$

where $\bar{*} v=\overline{* v}$. The curvature of $h$ is defined as an operator by

$$
\bar{\partial} \circ h^{-1} \circ \partial \circ h+h^{-1} \circ \partial \circ h \circ \bar{\partial}
$$

which we identify with the exterior multiplication by a $\operatorname{Hom}(E, E)$-valued $(1,1)$-form, the curvature form of $h$, denoted by $\Theta_{h}$. With respect to a local coordinate $\left(z^{1}, \ldots, z^{n}\right)$ of $M$ and a local fiber coordinate of $E, \Theta_{h}$ is locally expressed as

$$
\Theta_{h}=\left(\sum_{\alpha, \beta} \Theta_{\alpha \bar{\beta} \nu}^{\mu} d z^{\alpha} \wedge d \bar{z}^{\beta}\right)_{\mu, \nu} .
$$

$(E, h)$ and $\Theta_{h}$ are said to be Nakano semipositive, or semipositive in the sense of Nakano, if $h \Theta$ is semipositive as a Hermitian form on the fibers of $T_{M}^{1,0} \otimes E, T_{M}^{1,0}$ 
being the holomorphic tangent bundle of $M$. In terms of the local coordinates, this is equivalent to saying that the quadratic form

$$
\sum_{\alpha, \beta, \nu, \kappa}\left(\sum_{\mu} \Theta_{\alpha \bar{\beta} \nu}^{\mu} h_{\mu \bar{\kappa}}\right) \xi^{\alpha \nu} \bar{\xi}^{\beta \kappa}
$$

is semipositive. Here $h_{\mu \bar{\kappa}}$ is the local expression of $h$. In what follows we shall identify $\Theta_{h}$ with the curvature operator $\bar{\partial} h^{-1} \partial h+h^{-1} \partial h \bar{\partial}$ and denote the Nakano semipositivity simply by $\Theta_{h} \geq 0$. It is easy to see that $\Theta_{h} \geq 0$ if and only if

$$
\left\langle\sqrt{-1} \Theta_{h} \Lambda u, u\right\rangle \geq 0
$$

holds for any $u \in C_{0}^{n, 1}(M, E)$ and that (1) holds for any $(n, q)$-form if it holds for the $(n, 1)$-forms (cf. [S]).

We put $\bar{\vartheta}=-* \bar{\partial} *$ and define the operators $\vartheta_{h}$ and $\partial_{h}$ by $(\bar{\partial} u, v)=\left(u, \vartheta_{h} v\right)$ and $\left(\partial_{h} u, w\right)=(u, \bar{\vartheta} w)$, respectively. These operators are naturally extended so that they act on the spaces $K^{p, q}(M, E)$.

Since $g$ is Kählerian, Nakano's formula says

$$
\bar{\partial} \vartheta_{h}+\vartheta_{h} \bar{\partial}-\partial_{h} \bar{\vartheta}-\bar{\vartheta} \partial_{h}=\sqrt{-1}\left(\Theta_{h} \Lambda-\Lambda \Theta_{h}\right)
$$

(cf. [W]).

For any p-form $\theta$ on $M$, let $e(\theta)$ denote the exterior multiplication by $\theta$ from the left hand side, and let $e(\theta)^{*}$ be the adjoint of $e(\theta)$ with respect to $\langle$,$\rangle .$ Then

$$
\bar{\partial} e(\alpha)^{*}+e(\alpha)^{*} \bar{\partial}+\bar{\vartheta} e(\bar{\alpha})+e(\bar{\alpha}) \bar{\vartheta}=\sqrt{-1}(e(\partial \alpha) \Lambda-\Lambda e(\partial \alpha))
$$

holds for any $(0,1)$-form $\alpha$ (of class $\left.C^{1}\right)$ on $M$ (cf. [O-5, Lemma 1]).

Combining (2) with (3), for any $C^{2}$ positive function $\eta$ on $M$ one has

(4) $\bar{\partial} e(\eta) \vartheta_{h}+\vartheta_{h} e(\eta) \bar{\partial}-\partial_{h} e(\eta)(\bar{\vartheta})-(\bar{\vartheta}) e(\eta) \partial_{h}$

$$
\begin{aligned}
= & \sqrt{-1} e(\eta)\left(\left(\Theta_{h}-\operatorname{Id}_{E} \otimes e\left(\eta^{-1} \partial \bar{\partial} \eta\right)\right) \Lambda-\Lambda\left(\Theta_{h}-\operatorname{Id}_{E} \otimes e\left(\eta^{-1} \partial \bar{\partial} \eta\right)\right)\right) \\
& +e(\bar{\partial} \eta) \vartheta_{h}+\bar{\partial} e(\bar{\partial} \eta)^{*}+\bar{\vartheta} e(\partial \eta)+e(\partial \eta)^{*} \partial_{h} .
\end{aligned}
$$

Therefore, for any $u \in C_{0}^{n, q}(M, E)$, the equality

$$
\begin{aligned}
& \left\|\sqrt{\eta} \vartheta_{h} u\right\|^{2}+\|\sqrt{\eta} \bar{\partial} u\|^{2}-\|\sqrt{\eta} \bar{\vartheta} u\|^{2} \\
& \quad=\left(\sqrt{-1} \eta\left(\Theta_{h}-\operatorname{Id}_{E} \otimes e\left(\eta^{-1} \partial \bar{\partial} \eta\right)\right) \Lambda u, u\right)+2 \operatorname{Re}\left(e(\bar{\partial} \eta) \vartheta_{h} u, u\right)
\end{aligned}
$$

holds true. 
Let $L_{(2)}^{p, q}(M, E)\left(=L_{(2)}^{p, q}(M, E)_{g, h}\right)$ be the space of square integrable $E$ valued $(p, q)$-forms with respect to $g$ and $h$. Since $g$ is complete, the equality (5) carries over to the subspace

$$
D^{n, q}=\left\{u \in L_{(2)}^{n, q}(M, E) \mid \bar{\partial} u \in L_{(2)}^{n, q+1}(M, E) \text { and } \vartheta_{h} u \in L_{(2)}^{n, q-1}(M, E)\right\}
$$

if $\Theta_{h}-\operatorname{Id}_{E} \otimes \eta^{-1} \partial \bar{\partial} \eta$ is Nakano semipositive and $\eta+|\bar{\partial} \eta|$ is bounded. This fact, which is now classical, can be shown by approximating the form by compactly supported ones by multiplying the cut off functions of the form $\chi_{R}(x):=\chi\left(\operatorname{dist}\left(x_{0}, x\right) / R\right)(R>0)$, where $\chi$ is a $C^{\infty}$ real valued function on $\mathbb{R}$ satisfying $\chi(t)=1$ for $t<1$ and $\chi(t)=0$ for $t>2$, and $\operatorname{dist}\left(x_{0}, x\right)$ denotes the distance from a fixed point $x_{0} \in M$ to $x$ (cf. [A-V]).

Even if $\eta+|\bar{\partial} \eta|$ is not bounded we have still the following, which we shall need later.

Lemma 2.1. $\quad$ Suppose that $\eta$ is bounded and that there exists a constant $\varepsilon>0$ such that

$$
\eta \Theta_{h}-\operatorname{Id}_{E} \otimes \partial \bar{\partial} \eta-\varepsilon \operatorname{Id}_{E} \otimes \partial \eta \bar{\partial} \eta \geq 0
$$

holds everywhere. Then the equality (5) holds for all $u \in D^{n, q}$.

Proof. Given any $u \in D^{n, q}$, put $u_{k}=\chi_{k} u$ where $\chi_{k}$ is as above. Then it is easy to verify, by using the assumption (6), that a subsequence of $u_{k}$ converges to $u$ with respect to the graph norm of $\bar{\partial}+\vartheta_{h}$ Since $\operatorname{supp} u_{k}$ is compact, there exists a sequence $u_{k, l} \in C_{0}^{n, q}(M, E)$ such that $u_{k, l}$ converges to $u_{k}$ with respect to the graph norm of $\bar{\partial}+\vartheta_{h}$. For such $u_{k, l}, \bar{\vartheta} u_{k, l}$ also converges because $\left(\sqrt{-1}\left(\Theta_{h}-\operatorname{Id}_{E} \otimes e\left(\eta^{-1} \partial \bar{\partial} \eta\right)\right) \Lambda u, u\right) \geq 0$ by assumption. Therefore $u_{k}$ belongs to the domain of $\bar{\vartheta}$ so that (5) also holds for $u_{k}$. By (6) we have also that $2 \operatorname{Re}\left(e(\bar{\partial} \eta) \vartheta_{h} u_{k}, u_{k}\right) \rightarrow 2 \operatorname{Re}\left(e(\bar{\partial} \eta) \vartheta_{h} u, u\right)$ as $k \rightarrow \infty$. Hence by taking the limit of (5) for $u_{k}$ we obtain the conclusion.

\section{§3. Harmonic Forms on Weakly 1-complete Kähler Manifolds}

Let $M$ be a complex manifold equipped with a plurisubharmonic exhaustion function $\varphi . M$ is said to be $C^{k}$-pseudoconvex if $\varphi$ is of class $C^{k}$. $C^{0}$ pseudoconvex manifolds are simply said to be pseudoconvex. If $\varphi$ is allowed to have discontinuities, $M$ is called weakly pseudoconvex. $C^{\infty}$-pseudoconvex manifolds are called weakly 1-complete. (Not so much is known about the distinction between these classes.) 
In the sequel we assume for simplicity that $M$ is a weakly 1-complete manifold, although some of the results are extended to the weakly pseudoconvex case.

Let us fix a weakly 1-complete manifold $(M, \varphi)$ of dimension $n$ equipped with a complete Kähler metric $g$. (Note that every weakly 1-complete Kähler manifold admits a complete Kähler metric (cf. [N]). For any Hermitian holomorphic vector bundle $(E, h)$ over $M$ we put

$$
\mathcal{H}^{p, q}(E)\left(=\mathcal{H}^{p, q}(E)_{g, h}\right)=\left\{u \in L_{(2)}^{p, q}(M, E) \mid \bar{\partial} u=0 \text { and } \vartheta_{h} u=0\right\} .
$$

Let $(L, b)$ be a Hermitian holomorphic line bundle over $M$ which has a nonzero holomorphic section $s$. We shall first describe a condition on $(g, h, b)$ under which the inclusion $s \mathcal{H}^{n, q}(E) \subset \mathcal{H}^{n, q}(E L)$ holds true, by refining the arguments of Enoki $[\mathrm{E}]$ and Takegoshi $[\mathrm{Tg}]$.

Let $|s|$ (resp. $|s|_{\varphi}$ ) be the pointwise norm of $s$ with respect to the fiber metric $b\left(\right.$ resp. $\left.b e^{-\varphi}\right)$. Note that $-\partial \bar{\partial} \log |s|^{2}=\Theta_{b}$ outside $s^{-1}(0)$.

Let $\lambda: \mathbb{R} \rightarrow[0, \infty)$ be a $C^{\infty}$ convex increasing function such that $4|s|^{2}<$ $e^{\lambda(\varphi)}$. Then we put

$$
\eta\left(=\eta_{\varepsilon}\right)=-\log \left(|s|_{\lambda(\varphi)}^{2}+\varepsilon\right)+\log \left(-\log \left(|s|_{\lambda(\varphi)}^{2}+\varepsilon\right)\right)+\frac{1}{\varepsilon}
$$

for $0<\varepsilon<\frac{1}{4}$.

Then, under the curvature conditions that $\Theta_{h} \geq 0$ and $\Theta_{h}-\varepsilon_{0} \operatorname{Id}_{E} \otimes \Theta_{b} \geq 0$ for some $\varepsilon_{0}>0$, one can find $0<\varepsilon_{1}<\frac{1}{4}$ such that

$$
\eta \Theta_{h}-\operatorname{Id}_{E} \otimes \partial \bar{\partial} \eta \geq \operatorname{Id}_{E} \otimes \eta^{-2} \partial \eta \bar{\partial} \eta
$$

holds for $0<\varepsilon<\varepsilon_{1}$. This can be shown by differentiating $\eta$ (cf. [O-5]).

It is clear that $\eta$ is bounded and hence (7) implies (6).

In this situation we have (5) for all $u \in D^{n, q}(q \geq 0)$ by Lemma 1 . In particular, for any $u \in \mathcal{H}^{n, q}(E)_{g, h}$ we have, combining (5) with (7),

$$
\left(\sqrt{-1} \eta^{-2} \partial \eta \wedge \bar{\partial} \eta \wedge \Lambda u, u\right)=0
$$

which immediately implies that $\vartheta_{h *}(s u)=0$ holds for $h_{*}=h e^{-\lambda(\varphi)} b$ for all $q$ because $\eta$ is a function of $|s|_{\lambda(\varphi)}$.

Thus we obtain the following.

Proposition 3.1. $\quad$ Let $(M, \psi, g)$ be a weakly 1-complete Kähler manifold of dimension $n$, and let $(E, h),(L, b), s, \lambda$ be as above. Then there exists a 
complete Kähler metric $g_{*}$ on $M$ such that, for any $C^{\infty}$ convex increasing function $\nu, s \mathcal{H}^{n, q}(E)_{g_{*}, h e^{-\lambda(\varphi)}}$ is contained in $\mathcal{H}^{n, q}(E \otimes L)_{g_{*}, h e^{-\nu(\varphi)-\lambda(\varphi)} b}$ for all $q$.

Proof of Theorem 1.1. Let $\lambda$ and $g_{*}$ be as above, and let $u$ be any $\bar{\partial}$-closed $E$ - valued $(n, q)$-form on $M$ such that $s u=\bar{\partial} v$ for some $v$. Then we choose the above $\nu$ in such a way that $u \in L_{(2)}^{n, q}(M, E)$ and $v \in L_{(2)}^{n, q}(M, E \otimes L)$. Let $H u$ be the orthogonal projection of $u$ to $\mathcal{H}^{n, q}(E)$. Since $u-H u$ is in the closure of the image of $\bar{\partial}$, so is $s(u-H u)$. But $s H u \in \mathcal{H}^{n, q}(E \otimes L)$ by Proposition 3.1, so that $s H u$ coincides with the orthogonal projection of $s u$ to $\mathcal{H}^{n, q}(E, L)$, which must be equal to zero since $v \in L_{(2)}^{n, q-1}(M, E \otimes L)$. Therefore $H u=0$, so that $u$ represents a cohomology class contained in the closure of zero in $H^{q}\left(M, K_{M} \otimes E\right)$.

\section{$\S 4$. Proof of Theorem 1.2}

Let $\mathcal{I}$ and $\sigma$ be as in the statement of Theorem 1.2. In order to argue as in the proof of Theorem 1.1, we first identify the sheaf cohomology group $H^{q}\left(M, K_{M} \otimes E \otimes \mathcal{I}\right)$ with certain $\bar{\partial}$-cohomology group on $M \backslash \sigma^{-1}(0)$.

For that, let $g^{\prime}$ be a complete Kähler metric on $M \backslash \sigma^{-1}(0)$ such that, for any point $p \in M$ one can find a neighbourhood $U$ of $p$, a Hermitian metric $g_{U}$ on $U$, and a bounded $C^{\infty}$ function $\psi$ on $U \backslash \sigma^{-1}(0)$ satisfying $g^{\prime}>g_{U}$ and $g^{\prime}=\partial \bar{\partial} \psi$ on $U \backslash \sigma^{-1}(0)$. As such a metric $g^{\prime}$ one may take for instance a metric of the form $g+\partial \bar{\partial}\left(\sum_{\alpha} \rho_{\alpha}\left(\log \left(-\log \left\|f_{\alpha}\right\|\right)\right)^{-1}\right)$. Here $f_{\alpha}=\left(f_{\alpha 1}, \ldots, f_{\alpha m}\right)$ are systems of local generators of $\mathcal{I}$ and $\left\{\rho_{\alpha}\right\}_{\alpha}$ is a locally finite system of nonnegative $C^{\infty}$ cut off functions with $\left|\partial \rho_{\alpha}\right|_{g}+\left|\partial \bar{\partial} \rho_{\alpha}\right|_{g} \ll 1$ and $\sum_{\alpha} \rho_{\alpha}>0$ $\left(\left\|f_{\alpha}\right\|^{2}=\left|f_{\alpha 1}\right|^{2}+\ldots+\left|f_{\alpha m}\right|^{2}\right)$ (cf. [O-3]).

Then, by the $L^{2}$ vanishing theorem of Demailly [D-1] (cf. also [O-1]), one has

$$
H^{q}\left(M, K_{M} \otimes E \otimes \mathcal{I}\right) \simeq H_{(2), l o c}^{n, q}(M, E)_{g^{\prime}, h / \sigma} .
$$

Here $H_{(2), l o c}^{n, q}(M, E)_{g^{\prime}, h / \sigma}$ is defined as the quotient of

$$
\left\{u \in L_{(2), l o c}^{n, q}(M, E)_{g^{\prime}, h / \sigma} \mid \bar{\partial} u=0\right\}
$$

by $L_{(2), l o c}^{n, q}(M, E)_{g^{\prime}, h / \sigma} \cap \bar{\partial} L_{(2), l o c}^{n, q-1}(M, E)_{g^{\prime}, h / \sigma}$, where $L_{(2), l o c}^{n, q}(M, E)_{g^{\prime}, h / \sigma}$ stands for the space of measurable E-valued $(n, q)$-forms on $M \backslash \sigma^{-1}(0)$ which are square integrable on $U \backslash \sigma^{-1}(0)$ for all $U \Subset M$ with respect to $g^{\prime}$ and $h / \sigma$. 
Let us put

$$
\begin{aligned}
\mathcal{H}^{n, q}(E \otimes \mathcal{I})_{g^{\prime}, h / \sigma} & =\left\{u \in L_{(2)}^{n, q}(M, E)_{g^{\prime}, h / \sigma} \mid \bar{\partial} u=\vartheta_{h / \sigma} u=0\right\} \\
\mathcal{H}^{n, q}(E)_{g^{\prime}, h} & =\left\{u \in L_{(2)}^{n, q}(M, E)_{g^{\prime}, h} \mid \bar{\partial} u=\vartheta_{h} u=0\right\} .
\end{aligned}
$$

Then, similarly as in the proof of Theorem 1.1, it follows directly from the curvature assumption that

$$
\mathcal{H}_{g^{\prime}, h e^{-\nu(\varphi) / \sigma}}^{n, q}(E \otimes \mathcal{I}) \subset \mathcal{H}_{g^{\prime}, h e^{-\nu(\varphi)}}^{n, q}
$$

holds for any choice of $C^{\infty}$ convex increasing function $\nu$, by modifying in advance $h$ to $h e^{-\lambda(\varphi)}$ and $\sigma$ to $e^{-\lambda(\varphi)} \sigma$ if necessary.

Therefore, if $u$ represents an element in the kernel of $H^{q}\left(M, K_{M} \otimes E \otimes \mathcal{I}\right) \rightarrow$ $H^{q}\left(M, K_{M} \otimes E\right)$, its harmonic part must be zero for some $g^{\prime}$ and $\nu$. Therefore $u$ must be in the closure of zero in $H^{q}\left(M, K_{M} \otimes E \otimes \mathcal{I}\right)$.

\section{$\S 5 . \quad$ Application and Some Remarks}

We shall derive a relation between the curvature property of canonical bundles and the "size" of the support of the associated multiplier ideal sheaves as an application of Theorem 1.3. Finally we add some remarks on the relation of our results with the $L^{2}$ extension theory.

Definition 5.1. A holomorphic line bundle $L \rightarrow M$ is said to be pseudoeffective if there exists a $C^{\infty}$ fiber metric $b$ of $L$ and a locally integrable nonpositive function $\psi$ on $M$ satisfying

$$
\sqrt{-1}\left(\Theta_{b}+\partial \bar{\partial} \psi\right) \geq 0
$$

in the sense of $(1,1)$-current.

This notion is the analytic analogue of pseudoeffective divisors (cf. [DPS]). $b e^{-\psi}$ is then called a singular fiber metric of $L$.

Let $\mathcal{I}_{\psi}$ be the sheaf of ideals in $\mathcal{O}_{M}$ defined by

$$
\mathcal{I}_{\psi, x}=\left\{\left.f_{x}\left|\int_{U} e^{-\psi}\right| f\right|^{2}<\infty \quad \text { for some neighbourhood } U \text { of } x\right\} .
$$

$\mathcal{I}_{\psi}$ is coherent $(\mathrm{cf} .[\mathrm{Nd}])$.

Proposition 5.1. Let $L$ be a pseudoeffective line bundle over a weakly 1-complete manifold $(M, \varphi)$. Then there exists a $C^{\infty}$ fiber metric $b$ of $L$ and $a$ locally integrable function $\psi$ on $M$ such that 
(i) $\sup \psi \leq 0$

(ii) $\sqrt{-1}\left(\Theta_{b}+\partial \bar{\partial} \psi\right) \geq 0$

(iii) The inclusion $H^{0}\left(M, L^{k} \otimes \mathcal{I}_{k \psi}\right) \subset H^{0}\left(M, L^{k}\right)$ is an isomorphism for every $k \geq 1$.

Proof. Let $M_{j}=\{x \mid \varphi(x)<j\}$. Then one can find a $C^{\infty}$ fiber metric $b$ of $L$ and locally integrable functions $\psi_{j}$ on $M_{j}$ satisfying (i), (ii), (iii) for $M_{j}$ in such a way that $\psi_{j}$ is a decreasing sequence on each $M_{j_{0}}$ for $j_{0} \leq j$ (cf. [D-2, Proof of Proposition 8.4]). By taking a sufficiently rapidly increasing function $\lambda$, and replacing $b$ by $b e^{-\lambda(\varphi)}$, one may assume that $\psi_{j}<\psi_{j+1}+2^{-j}$ on $M_{j-1}$. Then the fiber metric $b e^{-\lambda(\varphi)}$ and the function inf $\psi_{j}$ satisfies the required properties.

The singular fiber metric $b e^{-\psi}$ as above is called an analytic Zariski decomposition of $L$ by Tsuji [Tj].

Proposition 5.2. $\quad$ Let $M$ be a holomorphically convex Kähler manifold with seminegative canonical bundle, and let $L$ be a pseudoeffective line bundle over $M$. Suppose that there exists an analytic Zariski decomposition be $e^{-\psi}$ of $L$ such that, for $M^{\prime}:=M \backslash \operatorname{supp}\left(\mathcal{O}_{M} / \mathcal{I}_{\psi}\right), \psi \mid M^{\prime} \in C^{\infty}$ and $\Theta_{b}+(1+\varepsilon) \partial \bar{\partial} \psi \geq 0$ on $M^{\prime}$ for some $\varepsilon>0$. Then $H^{0}\left(M, L / \mathcal{I}_{\psi} L\right)=0$. In particular $\operatorname{supp}\left(\mathcal{O}_{M} / \mathcal{I}_{\psi}\right)$ does not contain any isolated point.

Proof. Since $K_{M} \leq 0$ it follows from Theorem 1.3 that the restriction map $H^{0}(M, L) \rightarrow H^{0}\left(M, L / \mathcal{I}_{\psi} L\right)$ is surjective, whose image is zero by (iii). Hence $H^{0}\left(M, L / \mathcal{I}_{\psi} L\right)$ must be zero.

Proposition 5.3. $\quad$ Let $M$ be a holomorphically convex Kähler manifold whose canonical bundle is pseudoeffective and admits an analytic Zariski decomposition be $e^{-\psi}$ such that $\psi \mid M^{\prime} \in C^{\infty}$ and $\left\{\Theta_{b}+(1+\epsilon) \partial \bar{\partial} \psi\right\} \mid M^{\prime} \geq 0$ for some $\epsilon>0$. Then $H^{0}\left(M, K_{M}^{2} / \mathcal{I}_{\psi} K_{M}^{2}\right)=0$. In particular, $\operatorname{supp}\left(\mathcal{O}_{M} / \mathcal{I}_{\psi}\right)$ does not contain any isolated point.

Proof. Since $H^{0}\left(M, K_{M}^{2} \otimes \mathcal{I}_{\psi}^{2}\right)=H^{0}\left(M, K_{M}^{2}\right)$ by (iii), one has à fortiori $H^{0}\left(M, K_{M}^{2} \otimes \mathcal{I}_{\psi}\right)=H^{0}\left(M, K_{M}^{2}\right)$. On the other hand, by Theorem 1.3 we have the surjectivity of the restriction map $H^{0}\left(M, K_{M}^{2}\right) \rightarrow H^{0}\left(M, K_{M}^{2} / \mathcal{I}_{\psi} K_{M}^{2}\right)$. Therefore $H^{0}\left(M, K_{M}^{2} / \mathcal{I}_{\psi} K_{M}^{2}\right)=0$. 
In the $L^{2}$ extension theory, it is assumed that either $M$ is a weakly pseudoconvex Kähler manifold (cf. [D-2]) or $M$ admits a nowhere dense subset $X$ whose complement is Stein, such that $X$ is locally negligible with respect to $L^{2}$ holomorphic functions (cf. [O-5]). The latter is satisfied by a certain class of non-Kähler manifolds as Hopf manifolds $\mathbb{C}^{n} \backslash\{0\} / \boldsymbol{Z}(n \geq 2)$. An analogue of Theorem 1.2 is true also in this situation.

Theorem 5.1. $\quad$ Let $M$ be a weakly 1-complete manifold, let $(E, h)$ be a Hermitian holomorphic vector bundle over $M$, and let $\mathcal{I}$ be a coherent ideal sheaf over $M$. Suppose that $E$ and $\mathcal{I}$ satisfy the curvature condition as in Theorem 1.2 and that $M$ contains a nowhere dense closed subset $X$ such that $M \backslash X$ admits a complete Kähler metric which dominates a Hermitian metric of $M$ outside $X$, and that each point of $X$ has a coordinate neighbourhood $U$ such that every $L^{2}$ holomorphic function on $U \backslash X$ is holomorphically extendable to $U$. Then the kernel of the homomorphism

$$
\iota: H^{1}\left(M, K_{M} \otimes E \otimes \mathcal{I}\right) \rightarrow H^{1}\left(M, K_{M} \otimes E\right)
$$

is contained in the closure of zero. If moreover $M$ is holomorphically convex, then $\iota$ is injective.

The proof goes similarly as that of Theorem 1.2. Namely, if $u$ represents an element in the kernel of the homomorphism from $H^{1}\left(M, K_{M} \otimes E \otimes \mathcal{I}\right)$ to $H^{1}\left(M, K_{M} \otimes E\right)$, by restricting $u$ to $M \backslash X \backslash \operatorname{supp}\left(\mathcal{O}_{M} / \mathcal{I}\right)$ we take its harmonic representative with respect to the sum of the prescribed metric on $M \backslash X$ and the Levi form of some function on $M \backslash \operatorname{supp}\left(\mathcal{O}_{M} / \mathcal{I}\right)$, which is complete, and a suitable singular fiber metric of $E$ as before. If the curvature condition is satisfied, then we change the fiber metric as before in order that we can take the harmonic part of $u$ and conclude its nullity. Since $\operatorname{deg} u=1$, this proves that the harmonic part of $u$ with respect to the original metric on $M$ is also zero. (Remember that the $L^{2}$ condition for the $(n, 0)$-forms does not depend on the choice of the metrics on the manifolds.)

We note that the following is essentially contained in the generalized $L^{2}$ extension theorem in [O-5].

Theorem 5.2. $\quad$ Under the situation of Theorem 5.1, suppose that $M \backslash X$ is Stein, $\left(\operatorname{supp}\left(\mathcal{O}_{M} / \mathcal{I}\right), \mathcal{O}_{M} / \mathcal{I}\right)$ is nonsingular (i.e. reduced and smooth), and that $X$ does not contain any component of $\operatorname{supp}\left(\mathcal{O}_{M} / \mathcal{I}\right)$. Then the homomorphism $H^{1}\left(M, K_{M} \otimes E \otimes \mathcal{I}\right) \rightarrow H^{1}\left(M, K_{M} \otimes E\right)$ is injective.

We would like to add two remarks on $L^{2}$ extendability from singular varieties. 
Proposition 5.4. $\quad$ Let $D$ be a bounded pseudoconvex domain in $\mathbb{C}^{n}$, let $S$ be a closed complex analytic subset of some neighbourhood of $\bar{D}$ such that $\partial D$ contains no singular points of $S$, and let $S_{0}=S \cap D$. Then there exists a constant $C$ such that, for any $L^{2}$ holomorphic function $f$ on $S_{0}$, there exists an $L^{2}$ holomorphic function $\tilde{f}$ on $D$ satisfying $\tilde{f} \mid S_{0}=f$ and $\int_{D}|\tilde{f}|^{2} \leq C \int_{S_{0}}|f|^{2}$.

Proof. Let $\Sigma$ be the set of singular points of $S$ contained in $D$. Since $\Sigma$ is a finite set, one can find a constant $C_{k}$, for any $k \in \boldsymbol{N}$, such that for any $L^{2}$ holomorphic function $f$ on $S_{0}$ there exists a polynomial $P_{k}$ satisfying $\int_{D}\left|P_{k}\right|^{2} \leq C_{k} \int_{S_{0}}|f|^{2}$ and

$$
\int_{S_{0}}\left|f(z)-P_{k}(z)\right|^{2} \operatorname{dist}(z, \Sigma)^{-k} \leq C_{k} \int_{S_{0}}|f|^{2} .
$$

Here $\operatorname{dist}(z, \Sigma)$ denotes the euclidean distance between $z$ and $\Sigma$.

Therefore, to find a required extension $f$ with the $L^{2}$ norm controle, one has only to apply the $L^{2}$ extension theorem of [O-5] for $f(z)-P_{k}(z)$ by choosing a sufficiently large $k$ independently of $f$.

In contrast to this affirmative fact, the uniformity of the $L^{2}$ extension is lost if $\Sigma \neq \phi$, as the following example shows.

Counterexample. Let $D$ be the open unit ball $|z|^{2}+|w|^{2}<1$ in $\mathbb{C}^{2}$, let $S$ be defined by $z w=0$, and let $\varphi_{k}$ be a decreasing sequence of $C^{\infty}$ plurisubharmonic functions on $D$ converging pointwise to $\log |z-w|^{2}$. Then there exist no universal constants $C$ such that, for any $k$ and for any holomorphic function $f$ on $S_{0}$ such that $\int_{S_{0}} e^{-\varphi_{k}}|f|^{2}<\infty$, there exists a holomorphic extension $f_{k}$ of $f$ to $D$ satisfying $\int_{D} e^{-\varphi_{k}}\left|f_{k}\right|^{2} \leq C \int_{S_{0}} e^{-\varphi_{k}}|f|^{2}$. Infact, had there been such a constant $C$, the function $z(z-w) /(z+w)$ on $S_{0}$ would be holomorphically extendable to $D$ in such a way that the extension vanishes on $z=w$, which is clearly impossible.

\section{References}

[A-V] Andreotti, A. and Vesentini, E., Carleman estimates for the Laplace-Beltrami equation in complex manifolds, Publ. Math. Inst. Hautes Études Sci., 25 (1965), 81-130.

[C] Cartan, H., Séminaire: Théorie des fonctions de plusieurs variables, Paris 1951/52.

[D-1] Demailly, J.-P., Estimation $L^{2}$ pour l'opérateur $\bar{\partial}$ d'un fibré vectoriel holomorphe sémi-positif au déssus d'une variété kählerienne complète, Ann. Sci. École Norm. Sup., 15 (1982), 457-511.

[D-2] Demailly, J.-P., On the Ohsawa-Takegoshi-Manivel $L^{2}$ extension theorem, Progr. Math., 188 (2000), 47-82. 
[D-P-S] Demailly, J.-P., Peternell, T. and Schneider, M., Pseudo-effective line bundles on projective varieties, math. AG/0006025 (2000)

[D-M] Diederich, K. and Mazzilli, E., A remark on the theorem of Ohsawa-Takegoshi, Nagoya Math. J., 158 (2000), 185-189.

[E] Enoki, I., Kawamata-Vieweg vanishing theorem for compact Kähler manifolds, Proc. Taniguchi Symp. (ed. Mabuchi et al.), 1993, Marcel Dekker, 59-68.

[K] Kollár, J., Higher direct images of dualizing sheaves I, Ann. of Math., 123 (1986), 11-42.

[M] Manivel, L., Un théorème de prolongement $L^{2}$ de section holomorphes d'un fibré vectoriel, Math. Z., 212 (1993), 107-122.

[N] Nakano, S., On the inverse of monoidal transformation, Publ. RIMS, Kyoto Univ., 6 (1970/71), 483-502.

[Nd] Nadel, A. M., Multiplier ideal sheaves and existence of Kähler-Einstein metrics of positive scalar curvature, Ann. of Math., 132 (1990), 549-596.

[O-1] Ohsawa, T., Vanishing theorems on complete Kähler manifolds, Publ. RIMS, Kyoto Univ., 20 (1984), 21-38.

[O-2] - On the extension of $L^{2}$ holomorphic functions II, Publ. RIMS, Kyoto Univ., 24 (1988), 265-275.

[O-3] Hodge spectral sequence and symmetry on compact Kähler spaces, Publ. RIMS, Kyoto Univ., 23 (1987), 613-625.

[O-4] Math. Z., 219 (1995), 215-225.

[O-5] - On the extension of $L^{2}$ holomorphic functions $\mathrm{V}$ - effect of generalization, Nagoya Math. J., 161 (2001), 1-21.

[O-6] - On the extension of $L^{2}$ holomorphic functions VI - a limiting case, Contemp. Math. 332, Explorations in Complex and Riemannian geometry, J. Bland et al. (ed.) A.M.S. 2003, 235-239.

[O-T] Ohsawa, T. and Takegoshi, K., On the extension of $L^{2}$ holomorphic functions, Math. Z., 195 (1987), 197-204.

[S] Siu, Y.-T., Complex-analyticity of harmonic maps, vanishing and Lefschetz theorems, J. Differential Geom., 17 (1982), 55-138.

[Sk] Skoda, H., Morphismes surjectifs de fibrés vectoriels sémi-positifs, Ann. Sci. École Norm. Sup., 11 (1978), 577-611.

[Tg] Takegoshi, K., Higher direct images of canonical sheaves tensorized with semipositive vector bundles by proper Kähler morphisms, Math. Ann., 303 (1995), $389-416$.

[Tj] Tsuji, H. Analytic Zariski decomposition, Proc. of Japan Acad., 61 (1992), 161-163.

[Tn] Tankeev, S. G., On n-dimensional canonically polarized varieties and varieties of fundamental type, Izv. A. N. SSSE, Ser. Math, 35 (1971), 31-44.

[W] Wells, R. O. Jr., Differential Analysis on Complex Manifolds, 2nd ed., Grad. Texts in Math., 65, 1979, Springer, Berlin-Heidelberg-New York. 